\title{
Boosting QED and QCD bound states in the path integral formalism
}

\author{
Yu.A.Simonov \\ State Research Center \\ Institute of Theoretical and Experimental Physics, \\ Moscow, 117218 Russia
}

July 9, 2021

\begin{abstract}
Wave functions and energy eigenvalues of the path integral Hamiltonian are studied in Lorentz frame moving with velocity $v$. The instantaneous interaction produced by the Wilson loop is shown to be reduced by an overall factor $\sqrt{1-\left(\frac{v}{c}\right)^{2}}$. As a result one obtains the boosted energy eigenvalues in the Lorentz covariant form $E=$ $\sqrt{\mathbf{P}^{2}+M_{0}^{2}}$, where $M_{0}$ is the c.m. energy, and this form is tested for two free particles and for the Coulomb and linear interaction.

Using Lorentz contracted wave functions of the bound states one obtains the scaled parton wave functions and valence quark distributions for large $P$. Matrix elements containing wave functions moving with different velocities strongly decrease with growing relative momentum, e.g. for the time-like formfactors one obtains $F_{h}\left(Q_{0}\right) \sim$ $\left(\frac{M_{h}}{Q_{0}}\right)^{2 n_{h}}$ with $n_{h}=1$ and 2 for mesons and baryons, as in the "quark counting rule".
\end{abstract}

\section{Introduction}

Bound states in quantum field theories are usually considered in the c.m. system, however many practical applications need the corresponding wave functions in the arbitrarily moving system, e.g. in formfactors, reaction etc. 
On the theoretical side the rules of the special relativity can predict the frame dependence for the global characteristics, such as the total energy. However, for the internal characteristics e.g. wave functions, the general theory is still unable to give answers, since the frame dependence of the full dynamics includes the interaction term [1].

A part of this difficulty is connected to the standard formalism of relativistic bound state equations, based on the Bethe-Salpeter integral equations, which is a complicated and not always a rigorous instrument (problems of the relative time, spurious solutions and difficulty to implement nonperturbative effects).

The study of boost effects in this framework was done in [2, 3, 4, 5, and recently an analysis of boosted bound state equation for the ep system was presented in [6], while the $1+1 e^{+} e^{-}$case was considered in [7, 8].

Another approach (the $N$-quantum approach) was used in [9], while the WKB method (expansion in powers of $\hbar$ ) in [10].

A more convenient tool to study the effects of boosting would be a Hamiltonian with e.g. instantaneous interaction, derived from the quantum field action. However this line of reasoning has several complications. First of all, the usual methods of introducing interaction via particle exchange - exclude the nonperturbative mechanisms, which produce confinement in QCD [11. Secondly, the Minkowskian particle exchange automatically brings in the appearance of the new Fock sectors with a new created particle in addition to the basic two-particle content. A way to handle this problem was suggested recently in [7]. In a general case one obtains the matrix Hamiltonian for the Fock column wavefunction, and one needs a systematic way to cut off the higher states to concentrate on the lowest ones.

Moreover, one should have a simple way to disregard the spin degrees of freedom in the first approximation to make clear the dynamical mechanism of boosting.

All these problems are economically treated in the path integral (world line) formalism [12] based on the Fock-Feynman-Schwinger representation [13. Recently this formalism was presented in an especially useful form [14], where higher Fock state can be systematically eliminated, and the spindependent interaction treated separately [15. The accuracy of the first simple approximation in QCD and QED was checked in [16].

As we show below, this formalism provides one with the Hamiltonian of an arbitrarily moving system with internal interaction, given by the Lorentz invariant construction - the Wilson loop. The latter includes both nonper- 
turbavive and perturbative (particle exchange) interactions written first in the Euclidean space-time via field correlators and therefore does not produce higher Fock states, the latter appear due to perturbative quantum creation.

Below we are using the path-integral formalism of [14], which provides the Hamiltonian of a two-particle state in a moving coordinate system, where the total momentum $\mathbf{P}$ of the state is defined. Our formalism yields the Hamiltonian with additional parameters $\omega_{1}, \omega_{2}$, which play the role of the virtual particle energies, and the stationary point analysis of the state in terms of $\omega_{1}, \omega_{2}$, exact at large time interval of the system $T$, yields the energy $E$ of the moving state.

It is essential, that the interaction in the system is obtained in QCD and QED from the Wilson loop, which is gauge and Lorentz invariant, and where the resulting instantaneous (or light-cone) interaction is obtained via field correlators [14], see [17] for the corresponding light-cone work. In this way one can in principle define interaction in any moving system, however practically this task can be difficult. We are using general properties of the Wilson loop and make an ansatz for wave function. As a result one can find the approximate wave function and energy of a given state in a moving coordinate system.

One should stress, that the study of the boosted dynamics by itself has not much practical meaning, since all inertial systems are equivalent. However in practice there are many processes, which include interactions of slow and fast particles, and, hence, possible overlap integrals of their wave functions, and here the knowledge of boosted dynamics is necessary, and as we show, it leads to remarkable effects.

In the present paper we are addressing these problems for the QCD and QED bound states. We construct the Hamiltonian $H(\mathbf{P})$ of the two-body system and find its eigenvalues. We show that the eigenvalue in the moving system $E_{0}(\mathbf{P})$ can be found in the form

$$
E_{0}(\mathbf{P})=\sqrt{\mathbf{P}^{2}+\tilde{M}_{0}^{2}},
$$

where $\tilde{M}_{0}$ is defined by the boosted interaction $V_{\mathbf{P}}$ and should coincide with the c.m. energy $M_{0}$ provided $V_{\mathbf{P}}$ is found correctly.

Using the Wilson loop form, we find that the boost $L_{\mathbf{P}}$ acts on the original c.m. interaction at least in two ways: reorganizing its magnitude $V \rightarrow C V$, and changing its coordinate dependence

$$
V_{\mathbf{P}}=L_{\mathbf{P}} V=C V(L\{\mathbf{r}\})
$$


From the general Lorentz invariance of the Wilson loop one finds that at the stationary points

$$
C=C_{0}=\sqrt{1-\left(\frac{v}{c}\right)^{2}}=\frac{M_{0}}{E_{0}(\mathbf{P})} .
$$

Note, that we shall put $c=1$ in what follows. Neglecting at first the second modification $(V(\mathbf{r}) \rightarrow V(L(\mathbf{r})))$, we check that the mass $\tilde{M}_{0}$ in (1) obtained in the moving system approximately coincides with the original mass eigenvalue calculated the c.m. system. We consider several examples of $V(\mathbf{r}): 1$ ) linear confinement, 2) gluon or photon exchange and in both cases we find that $\tilde{M}_{0}$ agrees with $M_{0}$ within $10 \%$ or better.

To check the full Lorentz covariance of our procedure with $\omega_{1}, \omega_{2}$ we consider the relativistic two-body system of noninteracting particles of different masses $m_{1}, m_{2}$ and apply our procedure.

We find that indeed the eigenvalues $E_{0}(\mathbf{p})$ have the form (1) , where $\tilde{M}_{0}$ is the c.m. of two particles with the particle momenta $\mathbf{p}_{1}=\mathbf{p}, \mathbf{p}_{2}=-\mathbf{p}$ in the c.m. system, $\tilde{M}_{0}=M_{0}(\mathbf{p},-\mathbf{p})$. Having checked this (global) invariance, one must consider the case of the shape boosting - the so-called Lorentz-FitzGerald contraction [18, which is the result of the second step in the boosting transformation $V(\mathbf{r}) \rightarrow V(L(\mathbf{r}))$. This step cannot be obtained directly and kinematically since the instantaneous interaction in one system is not Lorentz (kinematically) connected to the instantaneous interaction in another moving system, and there occurs a serious restructuring of the interaction.

Therefore we impose the condition of the Lorentz contraction on the wave function and interaction, and in this way we find the explicit form of $L(\mathbf{r})$ in (2). This condition, which plays the role of an ansatz, defines the wave function in any system, provided it is known in the c.m. frame. As a result one can connect the Lorentz contracted wave function to the parton wave function at large $P$ and find, that it has usual scaling properties, but interconnected transverse and longitudinal dynamics. One also finds immediate consequences of Lorentz contraction for physical processes, e.g. for decay amplitudes, quark decay constants, form factors etc., part of which was discussed before (see e.g. [2] and [19]), while other should be investigated in future.

The paper is organized as follows. In the next section the general form of the path integral for two-body Green's function is written and the relativistic Hamiltonian is defined in terms of $\omega_{1}, \omega_{2}$ and Wilson loop interaction. 
In section 3 the case of the two noninteracting particles is considered and the general structure of Lorentz covariant eigenvalues $E_{n}=\sqrt{\mathbf{P}^{2}+M_{n}^{2}}$ is confirmed.

In section 4 the global property of the boosted interaction $V \rightarrow C V, C=$

$\frac{M_{n}}{E_{n}}$ is derived from the Wilson loop interaction and checked in the cases of Coulomb and confining interaction.

The section 5 is devoted to the Lorentz contraction ansatz and the ensuing properties of the wave function and interaction with possible consequences for physical effects. An appendix is devoted to the comparison of Lorentz contracted and scaled parton wave functions.

In the last section the main results and approximations are reiterated and some prospectives are given.

\section{The QCD and QED path-integral Hamilto- nian in the moving systems.}

The two-body $\left(q_{1}, \bar{q}_{2}\right)$ Green's function generated by the currents $j_{i}=\left(\psi \Gamma_{i} \psi\right)$ in the relativistic path formalism [14] can be written in the Euclidean spacetime

$$
G_{12}(x, y)=\frac{T}{2 \pi} \int_{0}^{\infty} \frac{d \omega_{1}}{\omega_{1}^{3 / 2}} \int_{0}^{\infty} \frac{d \omega_{2}}{\omega_{2}^{3 / 2}}\left(D^{3} z^{(1)} D^{3} z^{(2)}\right) \mathbf{x y}\left\langle\hat{T} W_{\sigma}(A)\right\rangle
$$

where $T$ is the Euclidean time interval, and

$$
\begin{gathered}
\langle\hat{T} W\rangle=4 \operatorname{tr} Y\langle W\rangle \exp \left(-K\left(\omega_{1}\right)-K\left(\omega_{2}\right)\right), \\
Y=\frac{1}{4} \Gamma_{1}\left(m_{1}-i \hat{p}_{1}\right) \Gamma_{2}\left(m_{2}-i \hat{p}_{2}\right)
\end{gathered}
$$

and

$$
K(\omega)=\int_{0}^{T} d t_{E}\left(\frac{\omega}{2}+\frac{m^{2}}{2 \omega}+\frac{\omega}{2}\left(\frac{d \mathbf{z}}{d t_{E}}\right)^{2}\right),
$$

while $\langle W\rangle$ is the Wilson loop, which can be expressed via field correlators

$$
\langle W(C)\rangle=\left\langle\operatorname{Tr} \exp i g \int d \pi_{\mu \nu}(z) F_{\mu \nu}(z)\right\rangle=\exp \sum_{n=1}^{\infty} \frac{(i g)^{n}}{n !} \int d \pi(1) \ldots d \pi(n)\langle\langle F(1) \ldots F(n)\rangle\rangle
$$




$$
d \pi(i)=d s_{\mu \nu}\left(z_{i}\right)+i \sigma_{\mu \nu} \frac{d t_{i}}{2 \omega_{i}} .
$$

As was shown in [1] the leading quadratic in $F$ term in the cumulant expansion (8) in the c.m. frame yields instantaneous confinement and gluon exchange interaction in the $3+1$ QCD case, and the Coulomb interaction in $3+1 \mathrm{QED}$

$$
\begin{gathered}
\langle W(C)\rangle=\exp \left\{-\int V\left(r\left(t_{E}\right)\right) d t_{E}+\text { spin dep. terms }\right\} \\
V_{Q C D}(r)=\sigma r-\frac{4}{3} \frac{\alpha_{s}}{r}, \quad V_{Q E D}(r)=-\frac{\alpha}{r} .
\end{gathered}
$$

It is clear that the vacuum averaging of $W(C)$, implied by the angular brackets, yields higher order corrections to $\alpha_{s}, e^{2}$ and $\sigma$.

We now turn to the case of the Hamiltonian in a moving Lorentz system. To this end we define the $q_{1} \bar{q}_{2}$ Green's function and Hamiltonian in the system with the total momentum $\mathbf{P}$ [14]

$$
\begin{aligned}
G_{P}(x, y)=\frac{T}{2 \pi} & \int_{0}^{\infty} \frac{d \omega_{1}}{\omega_{1}^{3 / 2}} \int_{0}^{\infty} \frac{d \omega_{2}}{\omega_{2}^{3 / 2}} Y d^{3}(\mathbf{x}-\mathbf{y}) e^{i \mathbf{P}(\mathbf{x}-\mathbf{y})} \times \\
& \times\left\langle\mathbf{x}\left|e^{-H\left(\omega_{1}, \omega_{2}, \mathbf{p}_{1}, \mathbf{p}_{2}\right) T}\right| \mathbf{y}\right\rangle
\end{aligned}
$$

with

$H\left(\omega_{1}, \omega_{2}, \mathbf{p}_{1}, \mathbf{p}_{2}\right)=\sum_{i} \frac{p_{i}^{2}+m_{i}^{2}+\omega_{i}^{2}}{2 \omega_{i}}+\hat{V}=\sum_{i=1,2} \frac{m_{i}^{2}+\omega_{i}^{2}}{2 \omega_{i}}+\frac{\boldsymbol{\pi}^{2}}{2 \tilde{\omega}}+\frac{\mathbf{P}^{2}}{2\left(\omega_{1}+\omega_{2}\right)}+\hat{V}$

Here we have replaced the instantaneous c.m. coordinates $\mathbf{x}_{1}$ and $\mathbf{x}_{2}$, by the relative and total c.m. coordinates

$$
\begin{gathered}
\boldsymbol{\rho}=\mathbf{x}_{1}-\mathbf{x}_{2}, \quad \mathbf{R}=\frac{\omega_{1} \mathbf{x}_{1}+\omega_{2} \mathbf{x}_{2}}{\omega_{1}+\omega_{2}}, \\
\boldsymbol{\pi}=\frac{\partial}{i \partial \boldsymbol{\rho}}, \quad \mathbf{P}=\frac{\partial}{i \partial \mathbf{R}}
\end{gathered}
$$

Note, that $\boldsymbol{\pi}$ is not the c.m. momentum, the latter we denote for two particles as $(\mathbf{p},-\mathbf{p})$. 
To have the lowest eigenvalue of $H\left(\omega_{1}, \omega_{2}, \mathbf{p}_{1}, \mathbf{p}_{2}\right)$ one should calculate the minimal value of its eigenvalue $E\left(\omega_{1}, \omega_{2}, \mathbf{P}\right)$ in the stationary value analysis with respect to $\omega_{1}, \omega_{2}$

$$
\left.\frac{\partial E\left(\omega_{1}, \omega_{2}, \mathbf{P}\right)}{\partial \omega_{i}}\right|_{\omega_{i}=\omega_{i}^{(0)}}=0, \quad i=1,2 ; \quad E_{0}(\mathbf{P}) \equiv E\left(\omega_{1}^{(0)}, \omega_{2}^{(0)}, \mathbf{P}\right)
$$

It is clear, that the instantaneous c.m. interaction $V_{c m}$ in (13) will be changed by the Poincare boost operator $\hat{L}(\mathbf{P})$ in the general system, hence one can write for $\hat{V}$ in (13)

$$
\hat{V}=\hat{L}(\mathbf{P}) V_{c m} .
$$

We expect that the eigenvalues of the operator (13) after minimization should have the form

$$
E_{0}(\mathbf{P})=\sqrt{\mathbf{P}^{2}+M_{0}^{2}}
$$

where $M_{0}$ is the c.m. energy of the $q_{1} \bar{q}_{2}$ system.

Note, that we have neglected for simplicity spin-dependent forces originating from $\sigma_{\mu \nu} \frac{d t}{2 \omega}$ in (9), see [15] for details and references. As a result our Hamiltonian is a $4 \times 4$ unit matrix in Dirac indices. We shall check (17) in the following chapters in the examples of free particles and QCD and QED systems.

\section{The case of two noninteracting particles}

In this case we define:

$$
\begin{gathered}
\Omega \equiv \omega_{1}+\omega_{2} ; \quad \omega_{1}=\Omega x, \quad \omega_{2}=\Omega(1-x) \\
H=\frac{\mathbf{P}^{2}+\Omega^{2}}{2 \Omega}+\frac{1}{2 \Omega} M_{0}^{2}, \quad M_{0}^{2} \equiv \frac{\boldsymbol{\pi}^{2}+m_{1}^{2}}{x}+\frac{\boldsymbol{\pi}^{2}+m_{2}^{2}}{1-x} .
\end{gathered}
$$

Minimizing $M_{0}^{2}$ in the c.m. system, where $\boldsymbol{\pi}=\mathbf{p}$ in the interval $x \in[0,1]$ one obtains the stationary point $x_{0}=\frac{\varepsilon_{1}}{\varepsilon_{1}+\varepsilon_{2}}$, where $\varepsilon_{i}=\sqrt{\mathbf{p}^{2}+m_{i}^{2}}$, and finally $M_{0}^{2}=\left(\varepsilon_{1}+\varepsilon_{2}\right)^{2}$, so that minimizing finally in $\Omega$, one has

$$
E_{0}(\mathbf{P})=\sqrt{\mathbf{P}^{2}+\left(\varepsilon_{1}+\varepsilon_{2}\right)^{2}} .
$$


Hence $\varepsilon_{1}+\varepsilon_{2}$ is the c.m.energy of two free particles with masses $\left(m_{1}, m_{2}\right)$ and momenta $(\mathbf{p},-\mathbf{p})$, and the Lorentz covariance of our expression is proved, if one assumes, that $M_{0}^{2}$ should be calculated in the c.m. system, where $\boldsymbol{\pi}=\mathbf{p}$.Now one has to find the same operator $M_{0}^{2}(19)$ in the moving system.

We consider again the Hamiltonian for the free particle motion, but now use Lorentz transformations for momenta to support the result (20)

$$
H_{0}=\frac{\mathbf{P}^{2}}{2\left(\omega_{1}+\omega_{2}\right)}+\frac{\omega_{1}+\omega_{2}}{2}+\frac{\boldsymbol{\pi}^{2}}{2 \tilde{\omega}}+\frac{m_{1}^{2}}{2 \omega_{1}}+\frac{m_{2}^{2}}{2 \omega_{2}},
$$

and all coordinates refer to the same moment of time, $\tilde{\omega}=\frac{\omega_{1} \omega_{2}}{\omega_{1}+\omega_{2}}$.

In terms of individual momenta in the moving system $\mathbf{p}_{i}=\frac{1}{i} \frac{\partial}{\partial z_{i}}$, one can write

$$
\mathbf{p}_{1}=\frac{\omega_{1}}{\omega_{1}+\omega_{2}} \mathbf{P}+\boldsymbol{\pi}, \quad \mathbf{p}_{2}=\frac{\omega_{2}}{\omega_{1}+\omega_{2}} \mathbf{P}-\boldsymbol{\pi}
$$

hence

$$
\mathbf{P}=\mathbf{p}_{1}+\mathbf{p}_{2}, \quad \boldsymbol{\pi}=\frac{\mathbf{p}_{1} \omega_{2}-\mathbf{p}_{2} \omega_{1}}{\omega_{1}+\omega_{2}} .
$$

Our next goal is to express $\boldsymbol{\pi}$ in terms of the relative c.m. momentum $\mathbf{p}(c . m.) \equiv \mathbf{p}$

Note, that the Hamiltonian (21) can be written as

$$
H_{0}=\frac{\mathbf{p}_{1}^{2}+m_{1}^{2}+\omega_{1}^{2}}{2 \omega_{1}}+\frac{\mathbf{p}_{2}^{2}+m_{2}^{2}+\omega_{2}^{2}}{2 \omega_{2}}
$$

and the on-shell condition for $\omega_{i}$ is obtained from the stationary point analysis of (24) in terms of $\omega_{1}$ and $\omega_{2}$. One has

$$
\omega_{i}^{(0)}=\sqrt{\mathbf{p}_{i}^{2}+m_{i}^{2}}, \quad i=1,2 .
$$

Hence $\omega_{i}^{(0)}$ plays the role of the particle energy and we shall below always replace $\omega_{i}$ by $\omega_{i}^{(0)}$, implying that both particles are on the mass shell. We shall show that the eigenvalues of the Hamiltonian (21) satisfy Eq. (11) and $\tilde{M}_{0}$ is exactly equal to the c.m. energy of two particles.

Introducing the velocity $v$ of the c.m. system, one can write

$$
\omega_{1}=\frac{\bar{\omega}_{1}+\mathbf{v p}}{\sqrt{1-v^{2}}}, \quad \omega_{2}=\frac{\bar{\omega}_{2}-\mathbf{v p}}{\sqrt{1-v^{2}}},
$$


where $\bar{\omega}_{1}$ are particle energies in the c.m. system. For parallel and perpendicular components of $\mathbf{p}_{i}$ w.r.t. $\mathbf{v}$ in terms of the c.m. momenta $p_{\|}, \mathbf{p}_{\perp}$ one has

$$
\begin{gathered}
p_{1}(\|)=\frac{p_{\|}+v \bar{\omega}_{1}}{\sqrt{1-v^{2}}}, \quad p_{2}(\|)=\frac{-p_{\|}+v \bar{\omega}_{2}}{\sqrt{1-v^{2}}}, \\
p_{1}(\perp)=p_{\perp}, \quad p_{2}(\perp)=-p_{\perp} .
\end{gathered}
$$

From (23) one obtains

$$
\boldsymbol{\pi}_{\perp}=\mathbf{p}_{\perp}, \quad \pi_{\|}=\frac{p_{\|}}{\sqrt{1-v^{2}}}+\frac{v}{\sqrt{1-v^{2}}}\left(\frac{\omega_{2} \bar{\omega}_{1}-\omega_{1} \bar{\omega}_{2}}{\omega_{1}+\omega_{2}}\right) .
$$

Inserting (26) in (29), one obtains

$$
\pi_{\|}=p_{\|} \sqrt{1-v^{2}}
$$

also $\omega_{1}+\omega_{2}=\frac{\bar{\omega}_{1}+\bar{\omega}_{2}}{\sqrt{1-v^{2}}}$.

As a result $H_{0}$ assumes the form

$H_{0}=\frac{\mathbf{P}^{2} \sqrt{1-v^{2}}}{2\left(\bar{\omega}_{1}+\bar{\omega}_{2}\right)}+\frac{\bar{\omega}_{1}+\bar{\omega}_{2}}{2 \sqrt{1-v^{2}}}+\frac{\sqrt{1-v^{2}}}{2}\left\{\frac{p_{\|}^{2}\left(1-v^{2}\right)+p_{\perp}^{2}+m_{1}^{2}}{\bar{\omega}_{1}+\mathbf{v p}}+\frac{p_{\|}^{2}\left(1-v^{2}\right)+p_{\perp}^{2}+m_{2}^{2}}{\bar{\omega}_{2}-\mathbf{v p}}\right\}$.

Using $\bar{\omega}_{i}^{2}=p_{\|}^{2}+p_{\perp}^{2}+m_{i}^{2}, \quad i=1,2$ one finally obtains

$$
H_{0}=\frac{\mathbf{P}^{2} \sqrt{1-v^{2}}}{2\left(\bar{\omega}_{1}+\bar{\omega}_{2}\right)}+\frac{\bar{\omega}_{1}+\bar{\omega}_{2}}{2 \sqrt{1-v^{2}}}+\frac{\bar{\omega}_{1}+\bar{\omega}_{2}}{2} \sqrt{1-v^{2}} .
$$

One can express $v$ as follows, $M_{0} \equiv \bar{\omega}_{1}+\bar{\omega}_{2}$,

$$
v=\frac{P}{\sqrt{P^{2}+M_{0}^{2}}}, \quad \sqrt{1-v^{2}}=\frac{M_{0}}{\sqrt{P^{2}+M_{0}^{2}}}
$$

and as a result one has

$$
H_{0}=\sqrt{\mathbf{P}^{2}+M_{0}^{2}}
$$

implying the Lorentz covariance of our approach.

It is now easy to compare our results for the free relativistic particles with that of the Newton-Wigner approach [20]. there the c.m. relative momentum $\mathbf{p}$ and the total momentum $\mathbf{P}$, defined as above in (22), (23), are connected with the momentum $\boldsymbol{\pi}=\frac{1}{i} \frac{\partial}{\partial \boldsymbol{\rho}}$ as follows:

$$
\mathbf{p}=\boldsymbol{\pi}+\frac{(\boldsymbol{\pi} \mathbf{P}) \mathbf{P}}{E_{0}\left(\sqrt{\mathbf{P}^{2}+E_{0}^{2}}+E_{0}\right)} .
$$


Here $\mathbf{p}$ and $\mathbf{P}$ are canonically conjugated to the relative and c.m. coordinates $\mathbf{x}$ and $\mathbf{X}$, defined as follows:

$$
\begin{gathered}
\mathbf{x}=\boldsymbol{\rho}+\frac{(\boldsymbol{\rho} \mathbf{P}) \mathbf{P}}{E_{0}\left(\sqrt{\mathbf{P}^{2}+E_{0}^{2}}+E_{0}\right)}+\frac{(\boldsymbol{\rho} \mathbf{P}) \mathbf{p}}{E_{0} \bar{\omega}_{1} \bar{\omega}_{2}}\left(\bar{\omega}_{1}-\bar{\omega}_{2}-\frac{\mathbf{p P}}{\sqrt{\mathbf{P}^{2}+E_{0}^{2}}}\right) \\
X_{i}=R_{i}-\frac{S_{i k} P_{k}}{E_{0}\left(\sqrt{\mathbf{P}^{2}+E_{0}^{2}}+E_{0}\right)}
\end{gathered}
$$

where

$$
S_{i k}=\rho_{i} \pi_{k}-\rho_{k} \pi_{i}
$$

One can use (33) to reduce (35) to the simple result

$$
p_{\|}=\pi_{\|} \frac{1}{\sqrt{1-v^{2}}}, \quad p_{\perp}=\pi_{\perp},
$$

which coincides with (29), (30). One can in principle express the total Hamiltonian in terms of canonically conjugated coordinates $\mathbf{x}, \mathbf{X}$ and momenta $\mathbf{p}, \mathbf{P}$ and proceed to solve with the interaction taken into account, which is a difficult road. Instead we shall investigate a simpler, however approximate way, where the boosted interaction is easier to implement.

\section{Boosting QCD and QED interaction}

We return to the general form (13) and discuss the Lorentz boost of the interaction. Note, that our result (13) is obtained in the moving Lorentz system. In particular, one can check, that $\int_{C} A_{\mu} d z_{\mu}$ as well as $\int_{S} d s_{\mu \nu} F_{\mu \nu}$ is not only gauge, but also Lorentz invariant structures.

Rewriting (10) as

$$
\langle W\rangle=\exp \left(-i \bar{V}(\mathbf{r}) T_{M}\right)
$$

one can exploit the Einsteinian time dilatation to predict, that any averaged interaction obtained from the Wilson loop average, transforms under Lorentz transformation from system $K^{\prime}$ to system $K^{\prime \prime}$, moving with respect to $K^{\prime}$ with velocity $v$ as follows

$$
\bar{V}^{\prime} T^{\prime}=\bar{V}^{\prime \prime} T^{\prime \prime}, \quad T^{\prime \prime}=\frac{T^{\prime}}{\sqrt{1-v^{2}}}, \quad V^{\prime \prime}=\bar{V}^{\prime} \sqrt{1-v^{2}} .
$$


This gives us the factor $C_{0}=\sqrt{1-v^{2}}$ announced in (3). However in the off-shell form of the Hamiltonian (13), before the stationary analysis in $\Omega$ is done, we shall use the off shell form for $C, C=\frac{M_{0}}{\Omega}$, which reduces to $C_{0}$ at the stationary point $\Omega=E_{0}$. Note, that this is not the only effect of the system motion, and we shall discuss the effect of the Lorentz contraction in section 5 .

Let us now use the property (41) to calculate the mass $M_{0}^{2}$ for different interactions. We shall write the Hamiltonian (13) as

$$
H=\frac{\mathbf{P}^{2}}{2 \Omega}+\frac{\Omega}{2}+\frac{\hat{M}_{0}^{2}}{2 \Omega}, \quad \frac{\hat{M}_{0}^{2}}{2 \Omega}=\frac{\boldsymbol{\pi}^{2}}{2 \tilde{\omega}}+C V(\mathbf{x})+\frac{m_{1}}{2 \omega_{1}}+\frac{m_{2}^{2}}{2 \omega_{2}} .
$$

We consider the cases of the Coulomb and linear interactions.

\section{1) The case of opposite charges in $3+1$ QED}

We now study eigenvalues of (13), where $\hat{V}(\mathbf{P})$ is given by (16) and we assume for simplicity, that $\hat{L}(\mathbf{P})$ may be represented by a simple factor, $\hat{L}(\mathbf{P}) \rightarrow C(\mathbf{P})$.

The eigenvalues of the equation

$$
\left(\frac{\pi^{2}}{2 \tilde{\omega}}-\frac{\alpha C}{r}\right) \varphi_{n}=\varepsilon_{n} \varphi_{n}
$$

are given by $\varepsilon_{n}=-\frac{\tilde{\omega}(\alpha C)^{2}}{2 n^{2}}$ and the total energy is (notations are as in (18) ),

$$
E\left(\omega_{1}, \omega_{2}, \mathbf{P}\right)=\frac{\mathbf{P}^{2}+\Omega^{2}}{2 \Omega}+\sum_{i=1,2} \frac{m_{i}^{2}}{2 \omega_{i}}-\frac{\tilde{\omega}(\alpha C)^{2}}{2 n^{2}} .
$$

Writing $E\left(\omega_{1}, \omega_{2}, \mathbf{P}\right)=E(\Omega, x, \mathbf{P})$, one obtains from the minimization in $x$,

$$
-\frac{m_{1}^{2}}{x^{2}}+\frac{m_{2}^{2}}{(1-x)^{2}}-\frac{(\Omega \alpha C)^{2}}{n^{2}}(1-2 x)=0
$$

For $m_{1}=m_{2}$ one obtains $x_{0}=1 / 2, \quad \omega_{1}^{(0)}=\omega_{2}^{(0)} \equiv \omega_{0}$

$$
E\left(\Omega, \frac{1}{2}, \mathbf{P}\right)=\frac{\mathbf{P}^{2}+\Omega^{2}}{2 \Omega}+\frac{2 m^{2}}{\Omega}-\frac{\Omega(\alpha C)^{2}}{8 n^{2}} \equiv \frac{\mathbf{P}^{2}+\Omega^{2}+\mathcal{M}_{0}^{2}}{2 \Omega} .
$$


Putting $C=\frac{\bar{M}}{\Omega}$, one finds the minimum of (46) varying in $\Omega$, which yields

$$
\begin{gathered}
E_{0}(\mathbf{P})=\sqrt{\mathbf{P}^{2}+\mathcal{M}_{0}^{2}}, \quad \Omega_{0}=\sqrt{P^{2}+\mathcal{M}_{0}^{2}}, \\
\mathcal{M}_{0}^{2}=4 m^{2}-\frac{\alpha^{2} \bar{M}^{2}}{4 n^{2}} .
\end{gathered}
$$

Inserting $\bar{M}=\mathcal{M}_{0}$ in (48) one obtains

$$
\mathcal{M}_{0}=\frac{2 m}{\sqrt{1+\frac{\alpha^{2}}{4 n^{2}}}} .
$$

This is exactly the spectrum found for the relativistic two-body Coulomb problem neglecting spin dependent and annihilation terms [16].

\section{2) The case of the linear confining potential in $3+1$ QCD.}

We are writing the Hamiltonian (13) in the form

$$
H=\frac{\mathbf{P}^{2}}{2 \Omega}+\frac{\Omega}{2}+\frac{1}{2 \Omega}\left(\frac{m_{1}^{2}}{x}+\frac{m_{2}^{2}}{1-x}+\frac{\boldsymbol{\pi}^{2}}{x(1-x)}+2 \bar{M} V(\mathbf{r})\right) \equiv \frac{\mathbf{P}^{2}+\mathcal{M}_{0}^{2}}{2 \Omega}+\frac{\Omega}{2}
$$

where we have denoted

$$
L(\mathbf{P}) V_{c m}(\mathbf{r})=C V_{c m}(\mathbf{r}), \quad C=\frac{\bar{M}}{\Omega},
$$

and $\bar{M}$ can in principle depend on $\mathbf{P}$ and $\boldsymbol{\pi}$, but we start with a simple ansatz with $\bar{M}=$ const.

For $m_{1}=m_{2}$ the minimization in $x$ yields $x_{0}=\frac{1}{2}$ and

$$
\mathcal{M}_{0}^{2}=4 m^{2}+\bar{\varepsilon}^{2}, \quad \varepsilon^{2} \varphi=\left(4 \boldsymbol{\pi}^{2}+2 \bar{M} V(\mathbf{r})\right) \varphi .
$$

For $V(\mathbf{r})=\sigma r$ the eigenvalues of (52) are known [21].

$$
\bar{\varepsilon}^{2}=(4 \bar{M} \sigma)^{2 / 3} a_{n}, \quad a_{0}=2.338, \quad \mathcal{M}_{0}=\sqrt{4 m^{2}+(4 \bar{M} \sigma)^{2 / 3} a_{n}} .
$$

Eqs. (52), (53) yield solution for $\mathcal{M}_{0}$ in the boosted system.

Let us now consider the massless case $m_{1}=m_{2}=0$ in (50) and take $\bar{M}=\mathcal{M}_{0}(c . m$.), which we shall calculate below separately. One has from (52), (53)

$$
\mathcal{M}_{0}^{2}=\left(4 \mathcal{M}_{0}(\text { c.m. }) \sigma\right)^{2 / 3} a_{n},
$$


while $\mathcal{M}_{0}($ c.m.) is found from (50) with $\mathbf{P}=0$ and $\bar{M}=\Omega$

$$
H(c . m)=\frac{\Omega}{2}+\frac{2 \pi^{2}}{\Omega}+\sigma r \rightarrow \mathcal{M}_{0}(c . m .)=\frac{\Omega}{2}+\left(\frac{2}{\Omega}\right)^{1 / 3} \sigma^{2 / 3} a_{n} .
$$

Minimizing $\mathcal{M}_{0}($ c.m.) in $\Omega$ one obtains the standard expression, commonly used in hadron spectra 21]

$$
\mathcal{M}_{0}(\text { c.m. })=4 \sqrt{\sigma}\left(\frac{a_{n}}{3}\right)^{3 / 4} .
$$

Insertion of (56) in (54) immediately yields

$$
\mathcal{M}_{0}=3^{1 / 2}(16)^{1 / 3} \sqrt{\sigma}\left(\frac{a_{n}}{3}\right)^{3 / 4} \approx 4.33 \sqrt{\sigma}\left(\frac{a_{n}}{3}\right)^{3 / 4} .
$$

One can see, that (56) and (57) coincide within the accuracy of $8 \%$, hence one can use approximately the following boosting factor of the linear potential

$$
L(\mathbf{P}) V_{c . m .}(r)=\frac{\mathcal{M}_{0}(c . m .)}{\Omega(\mathbf{P})} V_{c . m .}(r) .
$$

\section{Lorentz contraction and the boosted shape of a bound state in QCD and QED}

The full (infinite) set of all paths for the two-body Green's function (4) can be studied by making intersection with the $3 \mathrm{~d}$ hyperplane at some fixed time $t_{0}$. In this way one obtains the density of the state, given by the wave function $\Psi_{n}$

$$
\rho_{n}(\mathbf{x}, t)=\frac{1}{2 i}\left(\psi \frac{\partial}{\partial t} \psi^{+}-\psi^{+} \frac{\partial}{\partial t} \psi\right)=E_{n}\left|\Psi_{n}(\mathbf{x}, t)\right|^{2} .
$$

For a boosted coordinate system one can define the longitudinal and perpendicular sizes of the system $l_{\|}$and $l_{\perp}$

$$
\begin{aligned}
& l_{\|}=\int\left|x_{\|}\right| \rho_{n}(\mathbf{x}, t) d V \\
& l_{\perp}=\int\left|\mathbf{x}_{\perp}\right| \rho_{n}(\mathbf{x}, t) d V .
\end{aligned}
$$


Since densities obey the invariance law [18] under Lorentz transformations

$$
\rho(\mathbf{x}, t) d V=i n v
$$

and $x_{\|}$is Lorentz contracted in a moving system, one expects the following dependence of $l_{\|}, l_{\perp}$

$$
l_{\|}(v)=\sqrt{1-v^{2}} l_{\|}(0), \quad l_{\perp}(v)=l_{\perp}(0) .
$$

The basic question is how (63) is maintained and supported by the dynamics of the given system, i.e. what is the transformation law $L\{\mathbf{r}\}$ in

$$
L V(\mathbf{r})=C V(L\{\mathbf{r}\}) .
$$

Instead of solving this point for $V(\mathbf{r})$, we turn to the wave function $\Psi_{n}(\mathbf{x}, t)=$ $e^{-i E_{n} t} \varphi_{n}(\mathbf{r})$ and try to ensure the property (63) $)$.

One possible solution for $\varphi_{n}(\mathbf{r})$ to satisfy (60), (61) is

$$
L_{P} \varphi_{n}(r)=\varphi_{n}\left(x_{\perp}, \frac{x_{\|}}{\sqrt{1-v^{2}}}\right)
$$

On the other hand

$$
d V_{v}=\left(d x_{\|} d^{2} x_{\perp}\right)_{v}=\sqrt{1-v^{2}}\left(d x_{\|} d^{2} x_{\perp}\right)_{0}
$$

so that Eq. (62) is satisfied. Hence

$$
\begin{gathered}
\left(l_{\|}\right)_{v}=\int\left|x_{\|}\right| E_{n}\left|\varphi_{n}\left(x_{\perp}, \frac{x_{\|}}{\sqrt{1-v^{2}}}\right)\right|^{2}\left(d x_{\|} d^{2} x_{\perp}\right)_{v}= \\
=\left.\sqrt{1-v^{2}} \int \tilde{x}_{\|}\left|M_{0}\right| \varphi_{n}\left(x_{\perp}, \tilde{x}_{\|}\right)\right|^{2} d \tilde{x}_{\|} d^{2} x_{\perp}=\sqrt{1-v^{2}}\left(l_{\|}\right)_{0},
\end{gathered}
$$

where we have used $\tilde{x}_{\|}=\frac{x_{\|}}{\sqrt{1-v^{2}}}=x_{\|}^{(0)}$, i.e. the c.m. parallel coordinate. The next question is what kind of dynamics can ensure the transformation (65).

We now turn to our Hamiltonian

$$
H=\frac{\mathbf{P}^{2}}{2 \Omega}+\frac{\Omega}{2}+\frac{\left(h_{0}\right)_{v}}{2 \Omega},
$$

where

$$
\left(h_{0}\right)_{v}=\left(4 \boldsymbol{\pi}_{v}^{2}+4 m^{2}+2 \bar{M} V_{v}\right), \quad V_{v}=L V_{v}, \quad \boldsymbol{\pi}_{v}^{2}=L \boldsymbol{\pi}^{2}
$$


Note, that $\boldsymbol{\pi}$ is not canonical momentum and in general the transformation from the c.m. system with the instantaneous c.m. Hamiltonian $\left(h_{0}\right)_{\mathrm{cm}}$ to another instantaneous system with the total momentum $\mathbf{P}$ is not provided by Lorentz transformation. Therefore we start as before in section with the assumption that $\left(h_{0}\right)_{v}$ should be first solved in the c.m. system, and this result $\left(h_{0}\right)_{c m} \varphi_{c m}=M_{0}^{2} \varphi_{c m}$ should be inserted as an eigenvalue for $\left(h_{0}\right)_{v}$, i.e. $\left(h_{0}\right)_{v} \rightarrow\left(h_{0}\right)_{c m}=M_{0}^{2}$.

This assumption was proved to be true for the noninteracting pair in section 3 and approximately correct (within 10\%) for the typical interactions in section 4 . We now generalize this assumption to specify $\boldsymbol{\pi}_{v}^{2}$ and $V_{v}$, and to this end we define

$$
\left(h_{0}\right)_{v} \varphi_{v}=M_{0}^{2} \varphi_{v}, \quad \varphi_{v}=\varphi\left(x_{\|}^{v}, x_{\perp}^{v}\right)
$$

where

$$
\begin{gathered}
x_{\|}^{v}=\frac{x_{\|}}{\sqrt{1-v^{2}}}, \quad x_{\perp}^{v}=x_{\perp} ; \quad \pi_{\|}^{v}=\frac{1}{i} \frac{\partial}{\partial x_{\|}^{v}}, \\
\pi_{\perp}^{2}=\frac{1}{i} \frac{\partial}{\partial x_{\perp}^{v}}, \quad V_{v}=V\left(x_{\|}^{v}, x_{\perp}^{v}\right) .
\end{gathered}
$$

As a result the equation $\left(h_{0}\right)_{v} \varphi_{v}=m_{0}^{2} \varphi_{v}$ reduces to the c.m. equation $h_{0} \varphi=M_{0}^{2} \varphi$ by a simple change of variable $x_{\|}$with the same eigenvalue $M_{0}^{2}$. We now can write the boost transformations for $V$ and $\varphi_{n}$ as follows

$$
\begin{gathered}
V(r)=L V(X) \rightarrow C V\left(x_{\perp}, \frac{x_{\|}}{\sqrt{1-v^{2}}}\right), \quad C=\frac{M_{0}}{\Omega} \rightarrow \sqrt{1-v^{2}}, \\
\varphi_{n}(x) \rightarrow \varphi_{n}^{(v)}(x)=\varphi_{n}\left(x_{\perp}, \frac{x_{\|}}{\sqrt{1-v^{2}}}\right)
\end{gathered}
$$

with the normalization

$$
\int E_{n}\left|\varphi_{n}^{(v)}(x)\right|^{2} d V_{v}=1=\int M_{0}^{(n)}\left|\varphi_{n}^{(0)}(x)\right|^{2} d V_{0}
$$

As a result the shape of the object will be transformed according to the Lorentz contraction rule

$$
l_{\|}(v)=l_{\perp}(v) \sqrt{1-v^{2}}
$$


One of the immediate consequences of the form (74) is the high momentum asymptotics of the wave function

$$
\tilde{\varphi}_{n}^{(v)}(q)=\int \varphi_{n}^{(v)}(\mathbf{r}) e^{i \mathbf{q} \mathbf{r}^{3}} d^{3}=C_{0} \tilde{\varphi}_{0}\left(\mathbf{q}_{\perp}, q_{\|} \sqrt{1-v^{2}}\right),
$$

where $\tilde{\varphi}_{0}\left(q_{\perp}, q_{\|}\right)$is the Fourier transformed c.m. wave function. One can see in (76) , that in the case, when one of the constituents gets a large momentum $\mathbf{Q}\left(Q_{\|}, 0,0\right)$, the boosted wave function has a saturating limit up to the factor $C_{0}$ namely

$$
\begin{gathered}
\tilde{\varphi}_{n}^{(v)}(\mathbf{q}+\mathbf{Q})=C_{0} \tilde{\varphi}\left(\mathbf{q}_{\perp},\left(q_{\|}+Q_{\|}\right) \sqrt{1-v_{q}^{2}}\right)= \\
\longrightarrow \underset{Q}{\longrightarrow} \frac{M_{0}}{Q} \tilde{\varphi}\left(\mathbf{q}_{\perp}, Q \frac{M_{0}}{\sqrt{Q^{2}+M_{0}^{2}}}\right) \rightarrow \frac{M_{0}}{Q} \tilde{\varphi}\left(\mathbf{q}_{\perp}, M_{0}\right)
\end{gathered}
$$

As a second example we consider the process of the strong decay of a meson $M_{1}$ in the state $n$ into a pair of hadrons $M_{2}, M_{3}$, which can be both mesons or both baryons $B_{2}, B_{3}$. The corresponding amplitude can be written for the $B \bar{B}$ decay as $[22]\left(\left(\mathbf{p}^{\prime}, \mathbf{p}^{\prime \prime}\right)\right.$ are two relative momenta in the $3 q$ system $)$

$$
J_{n B \bar{B}}(\mathbf{p})=\int y_{\text {red }}^{B \bar{B}} \frac{d^{3} \mathbf{p}^{\prime}}{(2 \pi)^{3}} \frac{d^{3} \mathbf{p}^{\prime \prime}}{(2 \pi)^{3}} \Psi_{n}^{+}\left(\mathbf{p}-\mathbf{p}^{\prime}-\mathbf{p}^{\prime \prime}\right) \Psi_{B}\left(\mathbf{p}^{\prime}, \mathbf{p}^{\prime \prime}\right) \Psi_{\bar{B}}\left(-\mathbf{p}^{\prime},-\mathbf{p}^{\prime \prime}\right)
$$

and for the $M_{2} M_{3}$ decay as $[23,24]$.

$$
J_{n n_{2} n_{3}}(\mathbf{p})=\int y_{\text {red }}^{M \bar{M}} \frac{d^{3} \mathbf{q}}{(2 \pi)^{3}} \Psi_{n}^{+}(\mathbf{p}+\mathbf{q}) \Psi_{n_{2}}(\mathbf{q}) \Psi_{n_{3}}(\mathbf{q}),
$$

where $y_{\text {red }}$ contains the explicit decay parameters, which belong to the system at rest of the initial meson, see [22, 23, 24] for details.

It is important, that the boosted wave functions in both cases belong to the decay products, moving with momenta $(\mathbf{P},-\mathbf{P}),\left(\right.$ where $M_{1}=\sqrt{M_{2}^{2}+\mathbf{P}^{2}}+$ $\sqrt{M_{3}^{2}+\mathbf{P}^{2}}$, ) which grows as $M_{1}$, when $M_{1}$ lends to infinity.

As we have found in (76), the momentum dependent wave function acquires the factor $C_{i}=\sqrt{1-v_{i}^{2}}$ for each momentum, so that in (79) $\psi_{n_{i}}(\mathbf{q})=$ $C_{i} \varphi_{n_{i}}\left(\mathbf{q}_{\perp}, q_{\|} \sqrt{1-v_{i}^{2}}\right), i=2,3$ while in (78)

$$
\Psi_{B}\left(\mathbf{p}^{\prime}, \mathbf{p}^{\prime \prime}\right)=\left(1-v_{B}^{2}\right) \varphi_{B}\left(\mathbf{p}_{\perp}^{\prime}, \mathbf{p}_{\perp}^{\prime \prime}, p_{\|}^{\prime} \sqrt{1-v_{B}^{2}}, p_{\|}^{\prime \prime} \sqrt{1-v_{B}^{2}}\right)
$$




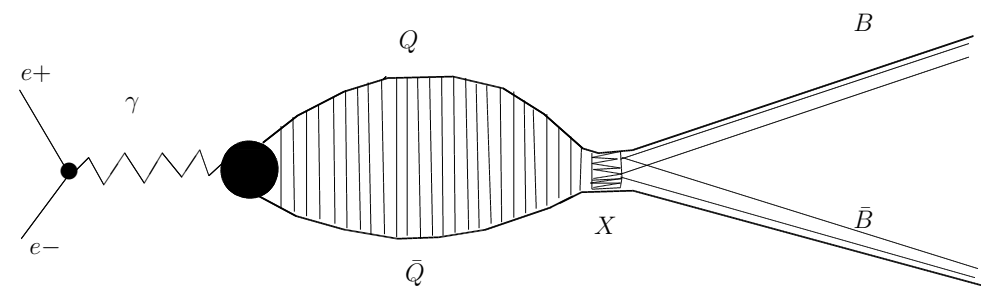

Figure 1: The amplitude of the process $e^{+} e^{-} \rightarrow B \bar{B}$ with an intermediate state $Q \bar{Q}(Q=u, d, s, \ldots)$. The step $X$ in the process is a nonperturbative double pair creation during a small time interval $\Delta t \sim \lambda \sim 0.1 \mathrm{fm}$.

As a result $J_{n}$ behaves as

$$
\begin{gathered}
J_{n B \bar{B}} \sim\left(1-v_{B}^{2}\right)^{2} \sim\left(\frac{M_{B}^{2}}{M_{B}^{2}+\mathbf{P}^{2}}\right)^{2} \\
J_{n n_{3} n_{3}} \sim \sqrt{\left(1-v_{2}^{2}\right)\left(1-v_{3}^{2}\right)} \sim\left(\frac{M_{2}^{2}}{M_{2}^{2}+\mathbf{p}^{2}} \frac{M_{3}^{2}}{M_{3}^{2}+\mathbf{P}^{2}}\right)^{1 / 2} .
\end{gathered}
$$

We can now turn to the processes $e^{+} e^{-} \rightarrow M_{2} M_{3}, e^{+} e^{-} \rightarrow B \bar{B}$ and the corresponding time-like formfactors, see Fig.1, and [22] for details.

To this end we define the cross section

$$
\sigma\left(e^{+} e^{-} \rightarrow h \bar{h}\right)=\frac{12 \pi \alpha^{2} p}{E^{3}}\left|\sum_{Q} e_{Q} \sum_{n} \frac{\Psi_{n}(0) \eta_{h Q} J_{n h h}(p)}{E_{n}-E+\frac{i \Gamma_{n}}{2}}\right|^{2}
$$

where $h=M, B$ and $\eta_{h Q}$ is the spin-recoupling coefficient and the time-like formfactor is proportional to (82), $F\left(Q_{0}\right) \sim \frac{\sigma E^{3}}{p \alpha^{2} 4 \pi}$ where $Q_{0}=E$

Note, that the sum over $n$ with the energy denominator has the same structure as in the total hadronic ratio $R$, and brings in a constant up to possible logarithmic terms, see e.g. [25] for a discussion.

Therefore one obtains the following asymptotics of the formfactor, for mesons and baryons

$$
F_{h}\left(Q_{0}\right) \sim\left(\frac{M_{h}^{2}}{M_{h}^{2}+\mathbf{p}^{2}}\right)^{n_{h}}, \quad h=M, B, n_{M}=1, \quad n_{B}=2 .
$$


Here $Q_{0}^{2}=4\left(M_{h}^{2}+\mathbf{p}^{2}\right)$. One can see, that the law (83) coincides with the famous "quark counting rule" for formfactors, found in 26].

Finally, one can connect our boosted wave function with the partonic function of the hadron, [27], which in the limit of high momentum $P$ can be written in the scaled form

$$
\Psi_{N}\left(\mathbf{p}_{1}, \ldots \mathbf{p}_{N}\right) \rightarrow \psi\left(\left\{\mathbf{p}_{\perp}^{(N)}\right\}, x_{1}, \ldots x_{N}\right)
$$

where $x_{i}=\frac{p_{\|}^{(i)}}{P}$, and $\Psi$ is normalized in the Lorentz invariant way. We show below in the appendix, that for two equal mass partons both functions are connected as (see Appendix for details)

$$
\psi_{2}\left(\mathbf{p}_{\perp}, x_{1}, x_{2}\right)=M_{0} \sqrt{x_{1} x_{2}}(2 \pi)^{-3 / 2} \tilde{\varphi}_{0}\left(p_{\perp}, M_{0}\left(x_{1}-\frac{1}{2}\right)\right)
$$

which implies, that $\tilde{\varphi}_{0}$ has a correct scaling limit and that transverse and longitudinal degrees of freedom are combined, e.g. for the $S$ wave one has $\tilde{\varphi}_{0}=\varphi\left(\sqrt{p_{\perp}^{2}+\left(x_{1}-\frac{1}{2}\right)^{2} M_{0}^{2}}\right)$ and $M_{0}$ is the c.m. mass of the hadron.

From(85) one can define the valence quark distribution function in the hadron $D_{h}^{q}\left(x, k_{\perp}\right)$, e.g. for a meson

$$
D_{h}^{q}\left(x, k_{\perp}\right)=\frac{E_{h}^{2}}{\varepsilon_{1} \varepsilon_{2}}\left|\psi_{2}\left(p_{\perp}, x\right)\right|^{2}=\frac{M_{0}^{2}}{(2 \pi)^{3}}\left|\tilde{\varphi}_{0}\left(p_{\perp}, M_{0}\left(x-\frac{1}{2}\right)\right)\right|^{2} .
$$

Note, that (86) does not show any singularities, as $\tilde{\varphi}_{0}$ is a monotonic Fourier transform of the static hadron wave function, and the corresponding quark distribution function $D_{h}^{q}(x)=\int d^{2} k_{\perp} D_{h}^{q}\left(x, k_{\perp}\right)$ is decreasing for large $\left|x_{1}-\frac{1}{2}\right|$ and has a maximum at $x=\frac{1}{2}$, and no peak around $x=0$. At this point one should remember, that we are treating the isolated valence Fock component without the multigluon Regge ladder Fock components, which bring in the expected singularity of $D_{h}^{q}(x)$ at $x$ small. Nevertheless $\tilde{\varphi}_{0}\left(k_{\perp}, k_{\|}\right)$ can be a good starting approximation for the perturbative process evolution.

One should stress again, that we have imposed the Lorentz contraction mechanism on the valence component only, while higher components ( wee partons) may create a more complicated picture.

Therefore our valence component could be called the "pure valence" component, which is different from the standard valence component, e.g. $u^{v}(x)=u(x)-\bar{u}(x)$, containing Regge ladder contribution, and it has no singularities at small $x$. 


\section{Conclusion and prospectives}

We have used path integral technic to calculate Hamiltonian and wave functions of the boosted system of two particles interacting via Wilson loop average. In doing so we have neglected all Fock components of the wave function except for the chosen $(q \bar{q})$ or $\left(e_{1} e_{2}\right)$.

This may be called a quenched approximation, but inclusion of higher Fock components is technically possible, see e.g. [28], [29]. We have proceeded in in the following steps:

1) We have used the Wilson loop interaction to prove, that it acquires due to the boost an overall reduction factor $C=\frac{M_{0}}{\Omega} \rightarrow \sqrt{1-v^{2}}$.

2) We have considered a free system of two relativistic particles with different masses $m_{1}, m_{2}$ and proved, that our Hamiltonian correctly describes the spectrum of the boosted systems, when the relative motion part $\left(h_{0}\right)$ is computed in the c.m. system.

3) Combining 1) and 2) we have checked that our boosted Hamiltonian $H=\frac{\mathbf{P}^{2}+\Omega^{2}+h_{0}}{2 \Omega}, \quad h_{0}=h_{k i n}^{r e l}+2 M_{0} V$ approximately (within $10 \%$ ) reproduces the boosted spectrum

$$
E(P)=\sqrt{\mathbf{P}^{2}+M_{0}^{2}}
$$

after the minimization in $\Omega$ is imposed on $H$. This check was done for the Coulomb and confining interaction.

4) We have used the Lorentz contraction phenomenon to formulate the "Lorentz contraction rule", by which the two-body wave function in its dependence on the relative instantaneous coordinate $r$ in the moving system, behaves as $\varphi\left(\mathbf{r}_{\perp}, \frac{r_{\|}}{\sqrt{1-v^{2}}}\right)$, where $v$ is the velocity of the system.

Correspondingly the Fourier transform of the wave function

$C \tilde{\varphi}\left(p_{\perp}, \sqrt{1-v^{2}}\left(p_{\|}+Q\right)\right)$ appears to be sensitive to large momentum transfer $Q$, only through the factor $C_{0}=\sqrt{1-v^{2}}$.

As a result we have shown, that the amplitude of the two-meson decay process with large decay energy is proportional to the product $\left[\left(1-v_{1}^{2}\right)(1-\right.$ $\left.\left.v_{2}^{2}\right)\right]^{1 / 2}$. Moreover, as a consequence, the time-like form factors of mesons and baryons behave as $\frac{1}{Q^{2}}$ and $\frac{1}{Q^{4}}$ respectively, i.e. the same way, as in the "quark counting rule".

5) We have connected our Lorentz contracted wave function with the scaled partonic function and found a smooth limit between them. In this way one can insist, that our nonperturbative wave function can be used in hard processes, which opens a wide field of applications. Note at this point, that 
the effect of Lorentz contracted wave functions was discussed before in the literature in different forms, see e.g. the first reference in [2] for a discussion and additional references, and application to the reaction $\mathbf{p} \overline{\mathbf{p}} \rightarrow \pi \pi$ in [19]. Within the framework of the "Lorenz contraction rule" the initial and final wave functions entering in the hard processes can be directly taken from the c.m. bound state wave function $\psi_{\mathrm{cm}}$. We also proved that the properly defined through $\psi_{c m}$ parton wave functions have correct scaling limits not depending on the boost momentum $\mathbf{P}$.

The approach presented above, calls for further study and applications in numerous processes, where boosted wave functions appear in initial and final states, e.g. in form factors, decay processes, etc.

The author is grateful to B.L.Ioffe and O.V.Kancheli for useful discussions. This research was supported by the RFBR grant 1402-00395.

\section{Normalization of boosted many particle states}

Appendix 1

We start with the normalization of the multiparton wave function 27

$$
E(P) \int \prod_{i=1}^{N} \frac{d^{3} p_{i}}{\varepsilon_{i}} \delta\left(P-\sum_{k=1}^{n} p_{k}\right)\left|\psi\left(p_{1}, \ldots p_{N}\right)\right|^{2}=1, \varepsilon_{i}=\sqrt{\mathbf{p}_{i}^{2}+m_{i}^{2}}
$$

which in the limit of large $P$ for $x_{k}=\frac{p_{\|}^{(k)}}{P}$ can be written as (fast moving partons)

$$
\int \prod d^{2} p_{\perp}^{(i)} \frac{d x_{i}}{x_{i}} \delta^{(2)}\left(\sum_{i=1}^{N} \mathbf{p}_{\perp}^{i}\right) \delta\left(1-\sum_{i=1}^{N} x_{i}\right)\left|\psi\left(p_{\perp}^{(i)}, x_{i}\right)\right|^{2}=1 .
$$

For two partons our wave function (74) is normalized as

$$
M_{0} \int\left|\tilde{\varphi}_{0}\left(p_{\perp}, p_{\|}\right)\right| \frac{d^{3} p}{(2 \pi)^{3}}=E(P)\left(1-v^{2}\right) \int \mid \tilde{\varphi}_{0}\left(k_{\perp},\left.k_{\|} \sqrt{1-v^{2}}\right|^{2} \frac{d^{3} k}{(2 \pi)^{3}}=1,\right.
$$

and the parton wave function $\psi\left(p_{1}, p_{2}\right)$ in terms of our $\varphi(k)$ is 


$$
\psi\left(p_{1}, p_{2}\right)=\sqrt{\frac{\varepsilon_{1} \varepsilon_{2} M_{0}^{2}}{E(P)(2 \pi)^{3}}} \tilde{\varphi}_{0}, \quad \sqrt{1-v^{2}}=\frac{M_{0}}{\sqrt{P^{2}+M_{0}^{2}}} .
$$

To express $k_{\perp}, k_{\|}$via parton momenta $p_{i}$ one can use (27); hence

$$
p_{\|}^{(1)}=k_{\|}+\frac{P}{M_{0}} \bar{\omega}_{1}=P x_{1}
$$

where $M_{0}=\bar{\omega}_{1}+\bar{\omega}_{2}$, (which is approximately true also for interacting partons), and for equal mass partons one has

$$
x_{1}=\frac{k_{\|}}{P}+\frac{\bar{\omega}_{1}}{M_{0}} \rightarrow \frac{k_{\|} \sqrt{1-v^{2}}}{M_{0}}+\frac{1}{2} .
$$

At large $P \gg M_{0}$, one has the scaling limit

$$
\psi\left(p_{\perp}^{(i)}, x_{i}\right)=\frac{M_{0}}{(2 \pi)^{3 / 2}} \sqrt{x_{1} x_{2}} \tilde{\varphi}_{0}\left(p_{\perp}, x_{1}-\frac{1}{2}\right) .
$$

In a similar way the multiparton wave function is connected to our wave function $\varphi\left(\boldsymbol{\pi}_{1}, \ldots \boldsymbol{\pi}_{N-1}\right)$ as

$$
\psi_{N}\left(p_{1}, \ldots p_{N}\right)=\frac{1}{(2 \pi)^{\frac{3}{2}(N-1)}} \prod_{i=1}^{N} \sqrt{M_{0} x_{i}} \varphi_{N}\left(p_{\perp}^{(i)}, M_{0}\left(x_{i}-\nu_{i}\right)\right) .
$$

One should stress again, that $\tilde{\varphi}_{0}$ has no on-shell singularities and there is no factorization in $p_{\perp}^{(i)}, x_{i}$, since at lowest angular momentum

$\tilde{\varphi}_{0} \sim \tilde{\varphi}\left(\sqrt{\left(p_{\perp}\right)^{2}+M_{0}^{2}\left(x-\frac{1}{2}\right)^{2}}\right)$.

\section{References}

[1] P.A.M. Dirac, Rev. Mod. Phys. 21, 392 (1949)

[2] Y.S.Kim and R.Zaou, Phys. Rev. D4, 1764 (1971)

S.R.Coleman, Annals Phys. (NY), 101, 239 (1976).

[3] D.Alba, H.W.Crater and L.Lusanna, J.Phys. A40, 9585 (2007). 
[4] M.G.Rocha, F.J.Llanes-Estrada, arXiv:0910.1448; D.Schuette, S.Villaba-Chavez, Eur. J. Phys. A44 (2010); W.N.Polyzou, arXiv:0908.1441.

[5] D.G.Currie, T.F.Jordan and E.C.G.Sudarshan, Rev.Mod. Phys. 35, 350 (1963).

[6] M.Järvinen, Phys. Rev. D71, 085006 (2005).

[7] D.Dietrich, P.Hoyer and M.Järvinen, Phys. Rev. D85, 1405016 (2012).

[8] M.Järvinen, Phys. Rev. D70, 065014 (2004).

[9] S.Cowen, arXiv:1107.0958.

[10] S.J.Brodsky and P.Hoyer, Phys.Rev. D83, 045026 (2011); arXiv: 0909.3045

[11] H.G.Dosch, Phys. Lett. B190, 177 (1987); H.G.Dosch and Yu.A.Simonov, Phys. Lett. B205, 339 (1988);

Yu.A.Simonov, Nucl. Phys. B307, 512 (1988); Phys. Usp.,39, 313 (1996).

[12] R.P.Feynman, Rev. Mod. Phys. 20, 367 (1948);

R.P.Feynman and A.R.Hibbs, Quartum Mechanics and Path Integrals, (Mc Graw-Hill, New York, 1965);

Yu.A.Simonov, Nucl. Phys. B307, 512 (1988).

[13] Yu. A. Simonov and J.A.Tjon, Ann. Phys. (N.Y) 228, 1 (1993); ibid 300, 54 (2002).

[14] Yu. A. Simonov, Phys. Rev. D88, 025028 (2013); arXiv:1303.4952.

[15] Yu. A. Simonov, Phys.Rev. D 88, 053004 (2013); arXiv:1304.0365.

[16] Yu. A. Simonov, Phys.Rev. D 90, 013013 (2014), arXiv: 1402.2162.

[17] A. Yu. Dubin, A. B. Kaidalov, and Yu. A. Simonov, Phys.Lett. B343, 310 (1995); V.L.Morgunov, V.I.Shevchenko and Yu.A.Simonov, Phys. Lett. B 416, 433 (1998). 
[18] H.A.Lorentz, Encyklopädie der Mathematischen Wissenschaften, Band V, art. 13,14, Leipzig, 1904;

W.Pauli, Theory of Relativity, Pergamon Press, N.Y., 1958.

[19] B.El-Bennich and W.M.Kloet, Phys. Rev. C68, 014003 (2003).

[20] T.D.Newton and E.P.Winger, Rev.Mod. Phys. 21, 400 (1949);

Yu. S. Kalashnikova and A. V. Nefediev, Phys. At. Nucl. 61, 785 (1998); ibid. 60, 1389 (1997).

[21] E.Eichten, K.Gottfried, T.Kinoshita, K.D.Lane and T.M.Yan, Phys. Rev. D 17, 3090 (1978); ibid D21, 203 (1980. Yu.A.Simonov in: QCD: Perturbative or Nonperturbative?, L.Ferreira, P.Nogueira and J.SilvaMarcos eds., Interscience, Singapore, 2000; hep-ph/9911237.

[22] Yu. A. Simonov, Phys. Rev. D 85, 105025 (2012), ibid. 125025 (2012), arXiv:1109.5545.

[23] V.D.Orlovsky and Yu.A.Simonov, Phys. Rev. D 84, 065013 (2011).

[24] A. M. Badalian V.D.Orlovsky and Yu.A.Simonov, Phys. At. Nucl. 76, 525 (2013).

[25] Yu.A.Simonov, Phys. At. Nucl. 65, 135 (2002); hep-ph/0109081; Phys. At. Nucl. 58, 107 (1993), hep-ph/9311247.

[26] V.A.Matveev, R.M.Muradyan and A.N.Tavkhelidze, Lett. Nuovo Cim. 7, 719 (1973);

S.J.Brodsky and G.R.Farrar, Phys. Rev. Lett. 31, 1153 (1973).

[27] R.P.Feynman, Photon-Hadron Interactions, W.A.Benjamin Inc. New York, 1972;

B.L.Ioffe, V.A.Khose, and L.N.Lipatov, Deep Inelastic Processes, NorthHolland, 1984;

F.J.Yndurain, The Theory of Quark and Gluon Interactions, Springer, 2006.

[28] A.B.Kaidalov, At the Frontier of Particle Physics, hep-ph/0103011;

M.Shifman ed. vol.1,603;

F.Gross, I.V.Musatov, Yu.A.Simonov, Phys. At. Nucl. 69, 699 (2006). 
[29] Yu.A.Simonov, Phys. At. Nucl. 67, 553 (2004); hep-ph/0306310;

Yu.A.Simonov, in "I.Ya.Pomeranchuck and physics at the turn of the Century", A.Berkov, N.Narozhny and L.Okun eds. World Scientific, Singapore, 2003; hep-ph/0310031. 